\title{
MicroRNA-19b-3p regulates nasopharyngeal carcinoma radiosensitivity by targeting TNFAIP3/NF-KB axis
}

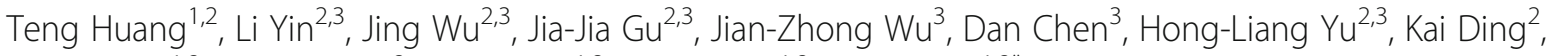
Nan Zhang ${ }^{1,2}$, Ming-Yu Dư ${ }^{2}$ Lu-Xi Qian ${ }^{1,2}$, Zhi-Wei Lu, ${ }^{1,2}$ and Xia He $e^{1,2^{*}}$

\begin{abstract}
Background: Nasopharyngeal carcinoma (NPC) is among the most common squamous cell carcinoma in South China and Southeast Asia. Radiotherapy is the primary treatment for NPC. However, radioresistance acts as a significant factor that limits the efficacy of radiotherapy for NPC patients. Growing evidence supports that microRNAs (miRNAs) play an important role in radiation response.
\end{abstract}

Methods: Real-time quantitative PCR was used to analyze the expression of miR-19b-3p in NPC cell lines and NP69. miR-19b-3p expression profiles in NPC tissues were obtained from the Gene Expression Omnibus database. The effect of miR-19b-3p on radiosensitivity was evaluated by cell viability assays, colony formation assays and in vivo experiment. Apoptosis and cell cycle were examined by flow cytometry. Luciferase reporter assay was used to assess the target genes of miR-19b-3p. Expression of target proteins and downstream molecules were analyzed by Western blot.

Results: miR-19b-3p was upregulated in NPC and served as an independent predictor for reduced patient survival. Radioresponse assays showed that miR-19b-3p overexpression resulted in decreased sensitivity to irradiation, whereas miR-19b-3p downregulation resulted in increased sensitivity to irradiation in vitro. Moreover, miR-19b-3p decreased the sensitivity of NPC cells to irradiation in vivo. Luciferase reporter assay confirmed that TNFAIP3 was a direct target gene of miR-19b-3p. Knockdown of TNFAIP3 reduced sensitivity to irradiation, whereas upregulation of TNFAIP3 expression reversed the inhibitory effects of miR-19b-3p on NPC cell radiosensitivity. Mechanistically, we found that miR-19b-3p increased NPC cell radioresistance by activating the TNFAIP3/ NF-KB axis.

Conclusions: miR-19b-3p contributes to the radioresistance of NPC by activating the TNFAIP3/ NF-KB axis. miR-19b$3 p$ is a determinant of NPC radioresponse and may serve as a potential therapeutic target in NPC treatment.

Keyword: microRNA-19b-3p, Nasopharyngeal carcinoma, Radiosensitivity, Apoptosis

\section{Background}

Nasopharyngeal carcinoma, which arises from the nasopharynx epithelium, is a common head and neck malignancy among the Southeast Asian populations. Radiotherapy is identified as the primary and only curative treatment for nasopharyngeal carcinoma [1].

\footnotetext{
* Correspondence: hexiaBM@163.com

${ }^{1}$ The Fourth Clinical School of Nanjing Medical University, Nanjing, Jiangsu, China

${ }^{2}$ Department of Radiation Oncology, Nanjing Medical University Affiliated Cancer Hospital, Cancer Institute of Jiangsu Province, 42 Bai Zi Ting Road, Nanjing, Jiangsu 210000, China

Full list of author information is available at the end of the article
}

Although advanced radiation technology is available, the relapse rate of nasopharyngeal carcinoma is as high as $82 \%$. Radioresistance is a major cause of treatment failure, leading to incomplete cure, recurrence, and metastasis [2].

miRNAs are a class of endogenous single-stranded small noncoding RNA molecules that act as negative post-transcriptional regulators of target gene expression [3]. Previous studies indicate that miRNAs directly affect radioresistance by interfering with specific pathways [4], including sensing DNA damage [5], repair of DNA double-strand breaks (DSBs) [6], cell cycle checkpoint 
activation [7], formation of reactive oxygen species [8] and apoptosis [9], and autophagy [10]. miR-19b-3p belongs to both the miR-17-92 and miR-106-363 clusters, which play significant roles in proliferation and cell survival $[11,12]$. In particular, miR-19b-3p has been validated as an oncogene in several human malignancies [12]. However, the mechanism underlying the role of miR-19b-3p in NPC radioresistance remains elusive.

Here, we report that miR-19b-3p was upregulated in NPC tissues and associated with worse patient prognosis. In addition, we confirmed that miR-19b-3p could affect the radiosensitivity of NPC cells by targeting the 3 '-UTR of TNFAIP3 and consequently activating the NF- $\mathrm{BB}$ pathway, leading to increased NPC radioresistance in vitro. miR-19b-3p possibly acts as a valuable target for subgroups of patients that might benefit from personalized therapeutic strategies.

\section{Methods}

\section{Patients and samples}

miR-19b-3p expression profiles in NPC tissues were obtained from Gene Expression Omnibus (GEO) (www.ncbi.nlm.nih.gov/geo/). Archived patient samples in The Cancer Genome Atlas (TCGA) database (https:// tcga-data.nci.nih.gov and https:// genomecancer.ucsc.edu) were selected. Exclusion criteria were as follows: 1) patients without radiation therapy; and 2) samples without completed data for analysis. Receiver operating characteristic (ROC) curve analysis was performed to determine cutoff score for differentiating between low and high miR-19b-3p levels among patients [13].

\section{Cell lines and culture conditions}

Five NPC cells (CNE1, CNE2, 5-8F, 6-10B and HNE1) and NP69 were originated from Research Center of Clinical Oncology of the Affiliated Jiangsu Cancer Hospital, Nanjing Medical University, Nanjing, China. The cells were propagated in RPMI 1640 medium (Hyclone, Logan, UT, USA) containing 10\% FBS (Gibco BRL, Gaithersburg, MD, USA) and 1\% antibiotic (Gibco BRL), and incubated at $37{ }^{\circ} \mathrm{C}$ with $\mathrm{CO} 2$ saturated.

\section{Irradiation}

Irradiation was delivered at room temperature using a $6 \mathrm{MeV}$ electron beam generated by a linear accelerator at a dose rate of $400 \mathrm{cGy} / \mathrm{min}$. A compensation glue with $1.0 \mathrm{~cm}$ thickness covered the cell culture containers. The source-to-skin distance was $100 \mathrm{~cm}$ [14].

\section{RNA sample preparation}

Total RNA was extracted using TRIzol reagent (Invitrogen, Burlington, ON, Canada) according to manufacturer's protocol. RNA yield and purity were determined by measuring the absorbance (Abs) at 260 and $280 \mathrm{~nm}$. Only RNA samples with Abs260nm/ Abs280nm ratio $>1.8$ were used.

\section{Cell transfection}

CNE-1 and CNE-2 cells were transfected with miR-19b-3p mimic/inhibitor/TNFAIP3-siRNA (RiboBio Guangzhou, China) according to manufacturer's protocol. To determine the efficiency of miRNA mimic/inhibitor/TNFAIP3-siRNA, the expression of miR-19b-3p/TNFAIP3 was assessed with a qRT-PCR detection system (Bio-Rad, Hercules, CA, USA) and Western blot apparatus.

\section{Quantitative reverse transcription-polymerase chain reaction analysis}

Total RNA was purified using TRIzol reagent (Invitrogen, Carlsbad, CA) according to manufacturer's instructions. Then, $2 \mu \mathrm{g}$ of total RNA was reverse-transcribed using Moloney murine leukemia virus reverse transcriptase (Promega, Madison, WI, USA) according to manufacturer's instructions. Expression levels of mature miRNAs were amplified using SYBR Green quantitative RT-PCR (qRT-PCR) on an ABI7300 real-time PCR machine (Applied Bio-systems). The expression of TNFAIP3 and $\beta$-actin was examined using the following specific primers: $5^{\prime}$-TGTGTATCGGTGCATGGTTTTA-3' and $5^{\prime}$-TCCTCAGGCTTTGTATTTGAGC-3' and $5^{\prime}$-G GACTTCGAGCAAGAGATGG-3' and 5'-AGCACTGT GTTGGCGTACAG-3', respectively. All reactions were performed in triplicate for each sample. Fold changes for miR-19b-3p and TNFAIP3 expression levels were calculated using the $2^{-\Delta \Delta \mathrm{Ct}}$ method.

\section{Cell viability assay}

Cell viability was actualized according to literature [15]. Cell counting kit-8 (Beyotime, China) was used according to the manufacturer's instructions to determine the growth curves for both cell lines. In brief, cells were cultured at $1.5 \times 10^{3}$ cells per well in triplicate in 96-well plates. Following a $12 \mathrm{~h}$ culture, the cells were exposed to various doses of radiation depending on the aim of the experiment. Absorbance values were expressed as percentages relative to the controls.

\section{Colony-forming assay}

Clonogenic survival assays were actualized according to literature [16]. Briefly, cells were plated in $60 \mathrm{~mm}^{2}$ culture dishes at various cell densities $\left(3 \times 10^{2}-2 \times 10^{3}\right.$ cells/well) for $12 \mathrm{~h}$ and were exposed to ionizing radiation (IR) in doses of $0,2,4,6$ and 8 Gy. The cells were 
cultured for an additional 8-12 days, and colonies were stained with Giemsa. Surviving colonies (a colony was defined as $>50$ cells) were counted. Radiation dose-response curves were created by fitting the data to the linear quadratic equation $S=e^{-\alpha \mathrm{D}-\beta \mathrm{D}^{\wedge} 2}$. Area under the curve (AUC) represents the mean inactivation dose (MID). The radiation protection factor (RPF) was calculated by dividing the MID of the test cells by the MID of control cells.

\section{In vivo experiment}

Six week old female nude mice were obtained from Medical Center of Yangzhou University. (Yangzhou, China). The nude mice were subcutaneously injected into the left inguinal with $5 \times 10^{6}$ CNE1 cells respectively. When the xenograft volumes reached $80 \mathrm{~mm}^{3}$, all mice were divided into 4 groups ( $n=6$ mice each), including miR-19b-3p agomir group (CNE1/miR-19b-3p agomir group), control agomir group (CNE1/ control agomir group), combination group 1 (CNE1/ miR-19b$3 \mathrm{p}$ agomir + RT group), combination group 2 (CNE1/ control agomir + RT group). $5 \mathrm{nmol} \mathrm{miR-19b-3p} \mathrm{agomir}$ or control agomir (RiboBio) in $50 \mu \mathrm{l}$ saline buffer was intratumorally injected into the tumor mass at multiple sites per mouse, and next day a 5 Gy dose of ionizing radiation was delivered to the combination group 1 and combination group 2. After 14 days, the combination group 1 and combination group 2 received another 5 Gy dose of ionizing radiation. $5 \mathrm{nmol}$ miR-19b-3p agomir or control agomir (RiboBio) was injected two times a week. All groups followed for about 4 weeks. Tumor volume (in $\mathrm{mm}^{3}$ ) was examined by caliper measurements every 3 to 4 days and calculated by using the modified ellipse formula (volume $=$ length $\times$ width $^{2} / 2$ ). Nude mice were sacrificed through decapitation as well as xenograft tumors were rapidly removed after death.

\section{Target prediction for miRNA candidates}

Identification of the predicted target mRNA genes of miRNA provides the basis for understanding miRNA functions. Thus, candidate target genes of miR-19b-3p were analyzed using the miRNA target prediction program PicTar (http://pictar.mdc-berlin.de/) and miRNA.org (http://www.microrna.org/microrna/home.do). Prediction values were calculated to estimate the binding affinities of the miRNAs and their predictive target genes.

\section{Western blot analyses}

After $48 \mathrm{~h}$ of transfection, cells were extracted and prepared in modified RIPA buffer (Beyotime, Shanghai, China). Total protein was extracted, and protein concentration was quantified using a BCA protein assay kit (Beyotime, Shanghai, China). A total of $20 \mathrm{mg}$ of protein from each sample was used for Western blot analysis. The primary antibodies used in this study included monoclonal anti-TNFAIP3 (1:1000; Abcam, HK), antiNF-kB/p- NF-kB (1:1000; Cell Signaling Technology, USA), anti-Bcl-2 (1:1000; Cell Signaling Technology, USA), anti-Bax (1:1000; Cell Signaling Technology, USA), and anti-capase-3/c-capase-3 (1:1000; Cell Signaling Technology, USA). $\beta$-actin was used as the loading control. Immunoreactive bands were visualized using ECL detection reagent (Millipore, Billerica, MA, USA). All data analyses were repeated thrice independently.

\section{Luciferase reporter construction and luciferase assays}

A segment of the human TNFAIP3 3'UTR region containing the predicted binding site for miR-19b-3p based on the TargetScan database (http://www.targetscan.org) was synthesized and inserted into the pCDNA3.1 luciferase reporter vector. The sequence of pri-miR-19b-3p also was inserted into the pCDNA3.1 plasmid. To generate the TNFAIP3 3'UTR mutated reporter, a number of nucleotides in the TNFAIP3 3'UTR that were complementary to the seed region nucleotides of miR-19b-3p were mutated. Cells of $293 \mathrm{~T}$ line were plated at a density of $1.5 \times 10^{6}$ cells in 24 -well plates 24 h before transfection. Reporter plasmids and miR-19b-3p expression plasmids were transfected using the Lipofectamine 2000 reagent (Invitrogen) according to manufacturer's protocol. The cells were lysed $48 \mathrm{~h}$ after transfection and their luciferase activity was assayed using the Picagene Dual SeaPansy luminescence kit (Toyo Ink, Tokyo, Japan) according to standard protocols [12]. All experiments were performed independently thrice. Luciferase activity was normalized to total protein concentration.

\section{Flow cytometric analysis of the cell apoptosis in response to irradiation}

After $36 \mathrm{~h}$ of transfection, CNE1 and CNE2 cells were seeded in 6-well plates $\left(5-7 \times 10^{5}\right.$ cells/well and each cell line had a parallel hole) for $12 \mathrm{~h}$ and three parallel holes were exposed to 6 Gy IR. Cells were harvested after culture for 3 days and washed twice with ice-cold PBS. Annexin V-FITC/PI staining was used to detect apoptotic cells. Three individual experiments were repeated.

\section{Pathway analysis}

The candidate target genes of miRNAs were analyzed using the miRNA target prediction program RNAhybrid (http://bibiserv.techfak.uni-bielefeld.de/rnahybrid/).

KEGG pathway analyses were performed to identify significant signal transduction pathways or enriched metabolic pathways in target gene candidates compared with the whole reference gene background. 


\section{Statistical analysis}

Student's $t$-test and one-way ANOVA statistical analyses were performed with GraphPad Prism 5.0 software and SPSS 13.0. Data were expressed as mean \pm standard deviation from at least three separate experiments. Differences were considered significant at $P<0.05$.

\section{Results}

miR-19b-3p is upregulated in NPC and associated with worse patient prognosis

miR-19b-3p expression profile was obtained using the GEO database for human nasopharyngeal carcinoma samples. Specifically, nasopharyngeal carcinoma $(n=62)$ expressed significantly more miR-19b-3p than normal nasopharyngeal samples $(n=6)$ (GSE36682) (Fig. 1a). The mRNA level of miR-19b-3p in five NPC cell lines (CNE1, CNE2, 5-8F, 6-10B, and HNE1) and NP69 were investigated by qRT-PCR (Fig. 1b). NP69 expressed lower levels of miR-19b-3p than the five NPC cell lines. Radiotherapy is identified as the primary treatment for NPC, but not much information is available on miR19b-3p's function in radioresistance. To further investigate the role of $\mathrm{miR}-19 \mathrm{~b}-3 \mathrm{p}$ in radioresistance, we analyzed data retrieved directly from TCGA. The cutoff value of miR-19b-3p was determined by receiveroperating characteristic analysis, which was used to differentiate between low and high miR-19b-3p levels among patients (Fig. 1c). Kaplan-Meier survival analysis of patients was performed based on the expression levels of miR-19b-3p and revealed that miR-19b-3p level negatively correlated with overall survival of patients (Fig. 1d). Univariate Cox regression analysis confirmed that miR-19b-3p expression level and lymph node classification significantly affected overall survival of

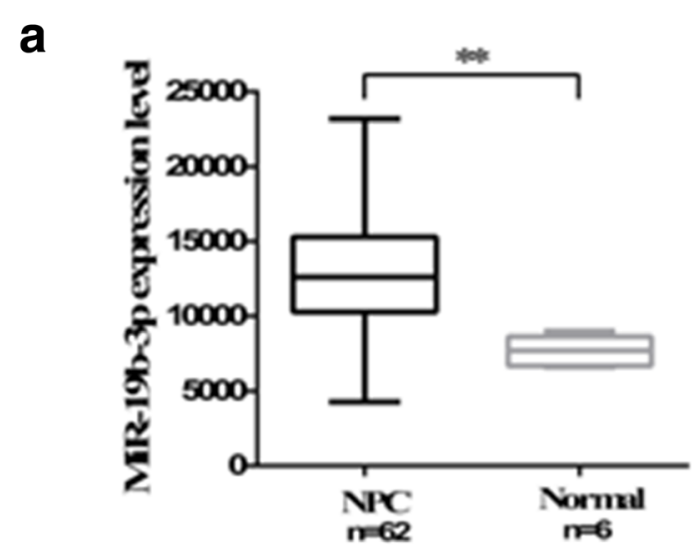

b
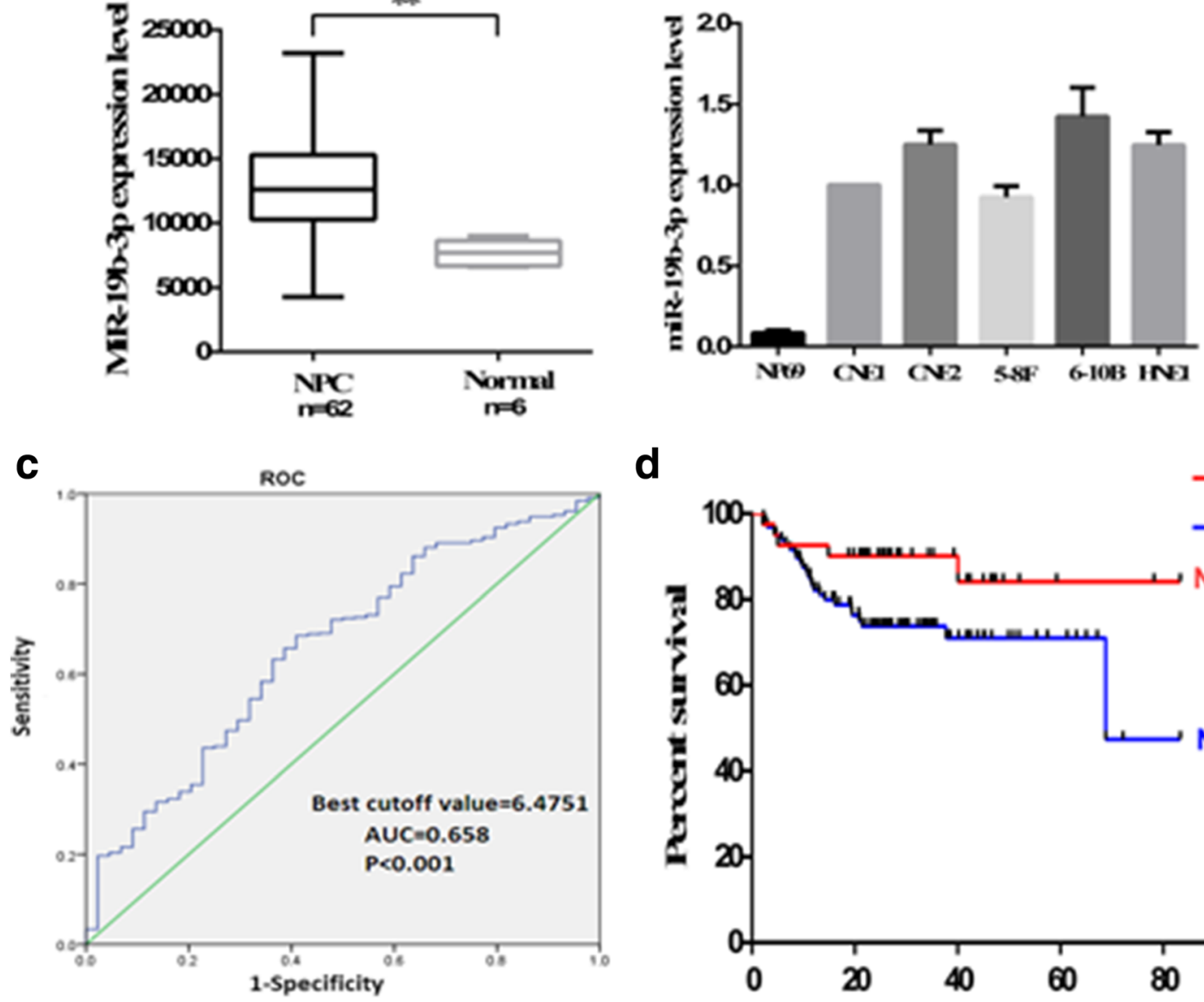

d

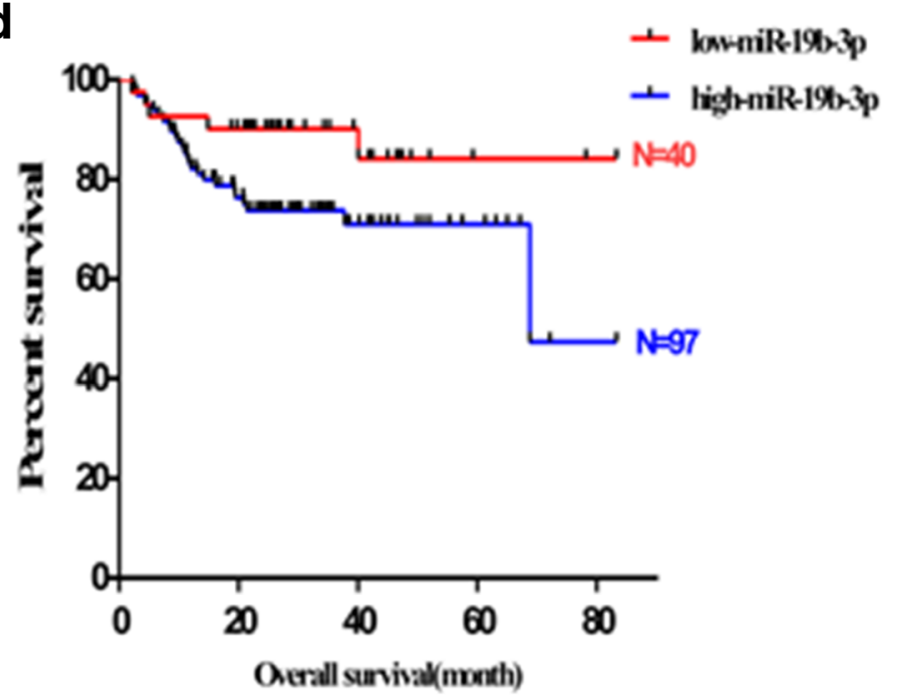

Fig. 1 Expression levels of miR-19b-3p in NPC and correlation of miR-19b-3p expression levels with patient survival. a Expression levels of miR-19b-3p in normal and NPC tissues. $\mathbf{b}$ Expression of miR-19b-3p was upregulated in NPC cells. $\mathbf{c}$ ROC analysis was performed to determine the cutoff value of miR19b-3p that could differentiate between the NPC patients with low and high miR-19b-3p levels $(P<0.001$; area under the ROC curve, 0.658 ; cutoff value, 6.4751). $\mathbf{d}$ Based on the expression levels of miR-19b-3p Kaplan-Meier survival analysis, patients with high miR-19b-3p expression had significantly worse overall survival than those with low miR-19b-3p expression. The log-rank test was used to calculate $p$ value 
patients. Multivariate Cox regression analysis showed that high miR-19b-3p expression was an independent predictor for the reduced overall survival of patients (Additional files 1 and 2) (Table 1). Taken together, these data indicate that miR-19b-3p possible play a significant role in NPC radioresistance.

\section{Ectopic expression of miR-19b-3p decreases sensitivity to irradiation}

To investigate the potential mechanism behind the role of miR-19b-3p in NPC radioresistance, we overexpressed miR-19b-3p in CNE-1 and CNE2 cells by transfected with miR-19b-3p mimic. QRT-PCR assays revealed that miR-19b-3p was efficiently upregulated $81.6 \pm 2.5$ times in CNE-1 cells and $132 \pm 5.29$ times in CNE2 (Fig. 2a). CCK-8 assay demonstrated increased survival rates of CNE-1 and CNE2 cells with miR-19b-3p overexpression (Fig. 2b). Moreover, clone survival assay showed that upregulation of miR-19b-3p expression markedly decreased radiosensitivity in the NPC cells [AUC 3.20 (CNE1 mimic) vs. 2.26 (CNE1.NC), RPF $=1.42$ AUC 2.86 (CNE2.mimic) vs. 2.51 (CNE2.NC), $\mathrm{RPF}=1.14 ; p<0.05$ ] (Fig. 2c, d). The overexpression of miR-19b-3p could significantly decrease the sensitivity of NPC cells.

Table 1 Univariate and multivariate analyses of prognostic factors for overall survival using Cox proportional hazards regression model $(N=137)$

\begin{tabular}{|c|c|c|c|c|}
\hline \multirow[t]{3}{*}{ Variable } & \multicolumn{4}{|c|}{ Overall survival } \\
\hline & \multicolumn{2}{|c|}{ Univariate analysis } & \multicolumn{2}{|c|}{ Multivariate analysis } \\
\hline & $\mathrm{HR}$ & $95 \% \mathrm{Cl}$ & $\mathrm{HR}$ & $95 \% \mathrm{Cl}$ \\
\hline \multicolumn{5}{|l|}{ Gender } \\
\hline Male & 1.507 & $0.617-3.68$ & 1.289 & $0.515-3.226$ \\
\hline Female & 1 & & 1 & \\
\hline \multicolumn{5}{|l|}{ Age (y) } \\
\hline$\leq 61$ & 0.898 & $0.444-1.819$ & 0.847 & $0.414-1.732$ \\
\hline$>61$ & 1 & & 1 & \\
\hline \multicolumn{5}{|c|}{ Primary tumor (T) stage } \\
\hline$T_{1}-T_{2}$ & 0.785 & $0.368-1.674$ & 0.759 & $0.351-1.641$ \\
\hline$T_{3}-T_{4}$ & 1 & & 1 & \\
\hline \multicolumn{5}{|c|}{ Lymph node $(\mathrm{N})$ classificaction } \\
\hline $\mathrm{N}_{0-1}$ & $0.418^{*}$ & $0.205-0.851$ & 0.532 & $0.252-1.124$ \\
\hline $\mathrm{N}_{2-3}$ & 1 & & 1 & \\
\hline \multicolumn{5}{|c|}{ Neoplasm histologic grade } \\
\hline $\mathrm{G}_{1}-\mathrm{G}_{2}$ & 0.739 & $0.348-1.572$ & 0.838 & $0.382-1.837$ \\
\hline $\mathrm{G}_{3}-\mathrm{G}_{4}$ & 1 & & 1 & \\
\hline \multicolumn{5}{|c|}{ Mir-19b-3p expression level } \\
\hline Low & $0.3^{*}$ & $0.104-0.863$ & $0.337^{*}$ & $0.114-0.992$ \\
\hline High & 1 & & 1 & \\
\hline
\end{tabular}

Inhibition of miR-19b-3p increases sensitivity to irradiation

Following miR-19b-3p overexpression in CNE-1 and CNE2 cells, we then suppressed miR-19b-3p expression in CNE1 and CNE2 cells. qRT-PCR confirmed that miR19b-3p expression was decreased to $62 \pm 0.6 \%$ in CNE1/ anti-miR-19b-3p cells and $76 \pm 0.85 \%$ in CNE2/ antimiR-19b-3p (Fig. 3a). CCK-8 assay revealed that the CNE1 and CNE2 cells with lower miR-19b-3p level had reduced survival capacity, following irradiation stimulation (Fig. 3b). Furthermore, clone survival assay showed that downregulation of miR-19b-3p expression increased radiosensitivity in the NPC cells [AUC 2.60 (CNE1.inhibitor) vs. 3.16 (CNE1.NC), RPF =0.82 AUC 2.92 (CNE2.inhibitor) vs. 3.32 (CNE2.NC), $\mathrm{RPF}=0.88$; $p<0.05$ ] (Fig. 3c and $\mathrm{d}$ ). Taken together, these data confirm that knockdown of miR-19b-3p could decrease the resistance of NPC cells to irradiation.

\section{miR-19b-3p decreased the sensitivity of NPC cells to irradiation in vivo}

The in vitro results revealed that overexpression of miR19b-3p can influence radiosensitivity in NPC cells, we furtherly investigated whether miR-19b-3p has a similar effect in vivo. Nude mice were injected with CNE1 cells. We found that miR-19b-3p did not affect tumor growth and weight of group without IR ( $p=0.31, p=0.79)$, but could significantly increase tumor growth and weight compared to that in miR-control group after exposure to irradiation $(p=0.0011, p=0.0073)$ (Fig. 4). These results are consistent with our in vitro results.

\section{miR-19b-3p depressed apoptosis after irradiation stimulation}

To investigate the potential role of miR-19b-3p in NPC radiobiology, pathway analysis of miR-19b-3p was performed (Fig. 5a). Results showed that miR-19b-3p may play a role in several radioresistance-associated pathways, such as NF-kB, Wnt, and P53 signaling pathways, which are closely related to apoptosis and cell cycle. Thus, both apoptotic and cell cycle effects of miR-19b$3 p$ were assessed by flow cytometry. We found that miR-19b-3p overexpression significantly decreased the percentage of apoptosis in CNE1 and CNE2 cells after irradiation relative to controls. Conversely, CNE1 and CNE2 cells with reduced miR-19b-3p significantly increased the percentage of apoptosis (Fig. $5 \mathrm{~b}$ and $\mathrm{c}$ ). However, miR-19b-3p had no effect on the cell cycle of the NPC cells after irradiation stimulation. The correlation between cell cycle and radioresistance in miR-19b3p may be diminished because of cell arrest after irradiation [17] (Fig. 5d). 
a

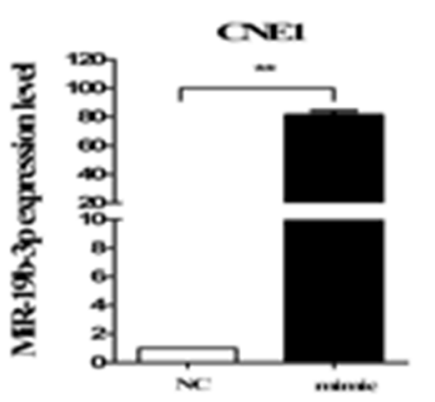

b

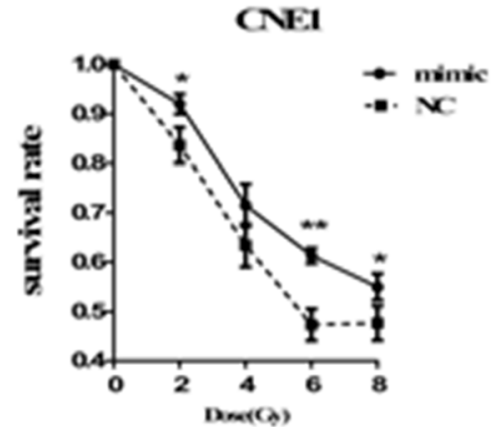

C

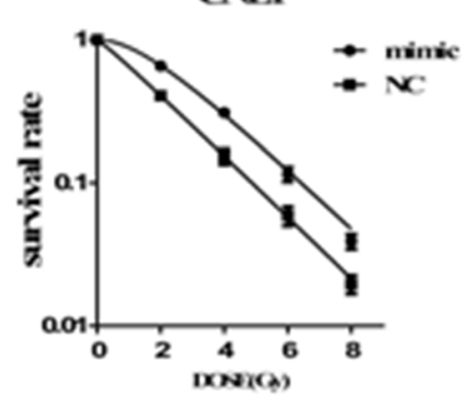

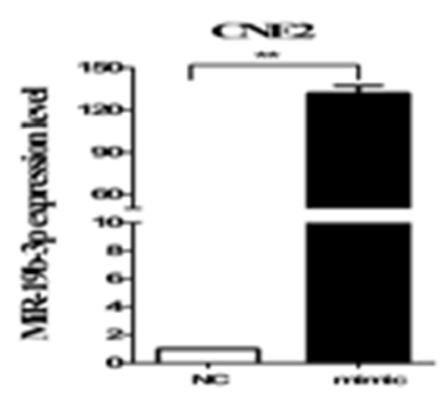

CNF2

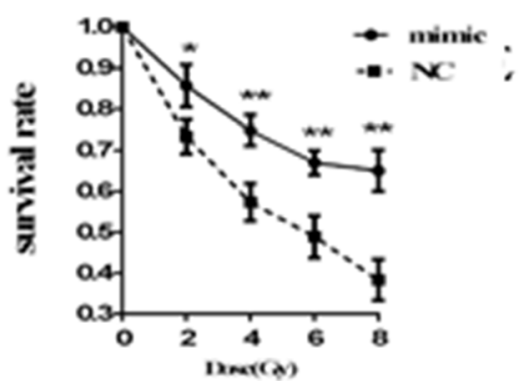

CN2

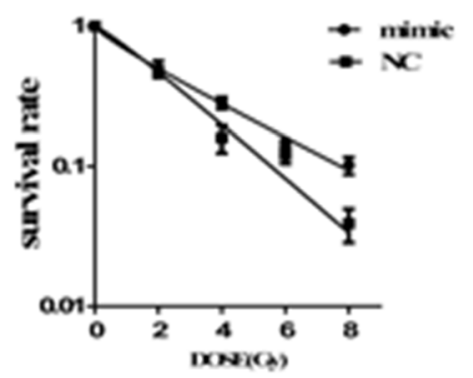

0Gy $2 \mathrm{~Gy} 4 \mathrm{~Gy} 6 \mathrm{~Gy} 8 \mathrm{~Gy}$

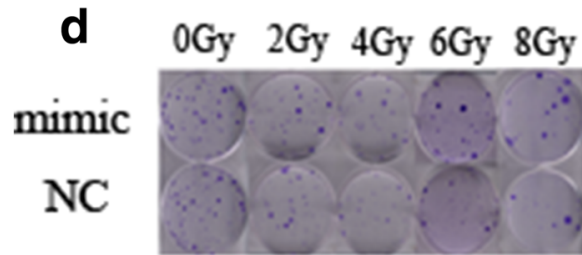

CNE1

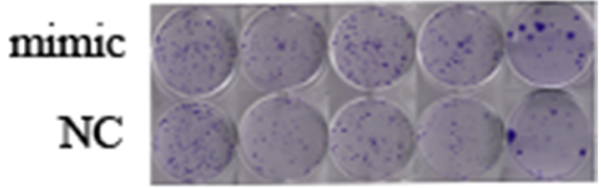

CNE2

Fig. 2 miR-19b-3p increased radioresistance of NPC cells. a miR-19b-3p upregulated expression in transfected cells. b Survival rates of different cell groups were examined using CCK-8 assays in CNE-1 and CNE-2 cells after 2,4,6,8 Gy irradiation. c A clonogenic survival assay shows that transfection of miR-19b-3p mimic increased NPC cells radioresistance compared with control group after 2,4,6,8 Gy irradiation. $\mathbf{d}$ A representative image of colony formation in CNE-1 and CNE-2 cells exposed to different doses of IR

miR-19b-3p regulates TNFAIP3 expression by targeting the 3'-UTR of TNFAIP3

Identification of the predicted target genes of miRNA provides the basis for understanding miRNA functions. Candidate miR-19b-3p target genes were analyzed using miRNA target prediction websites miRNA.org (www.microrna.org/microrna/home.do) and PicTar (http://pictar.mdc-berlin.de/) (Fig. 6a). We identified
TNFAIP3 as a candidate target gene, which includes a seed sequence at the 3 'UTR region that is complementary to a sequence within the $5^{\prime}$ end of miR-19b-3p. Moreover, we obtained the expression of miR-19b-3p and TNFAIP3 in 516 head and neck squamous carcinoma tissues from TCGA. Negative correlation between miR-19b-3p and TNFAIP3 was also observed (Additional file 3) (Fig. 6b). In addition, the TNFAIP3 mRNA regions complementary 


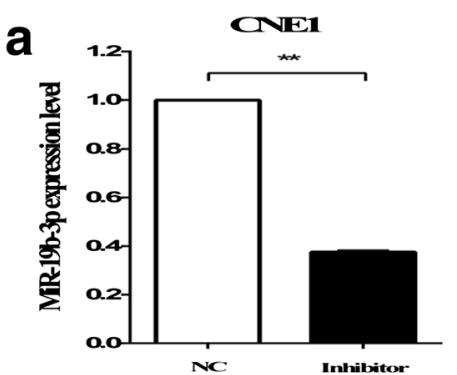

b

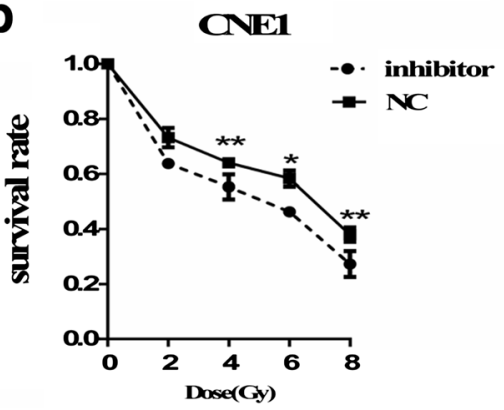

C
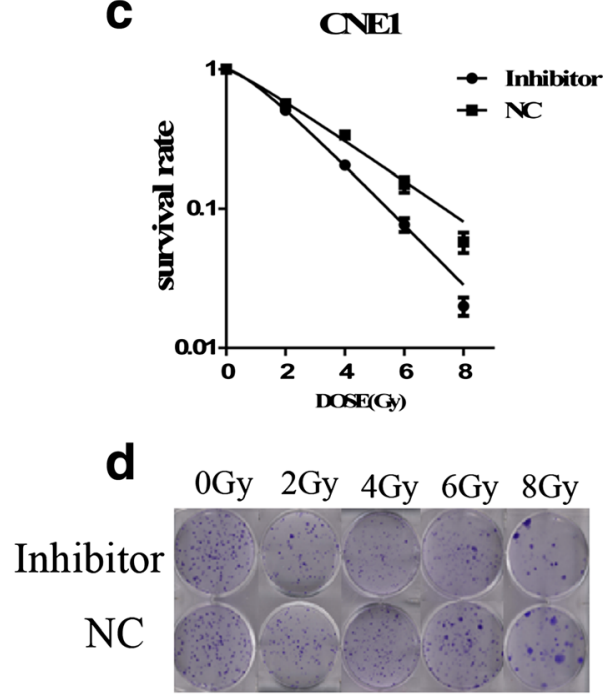

CNE1
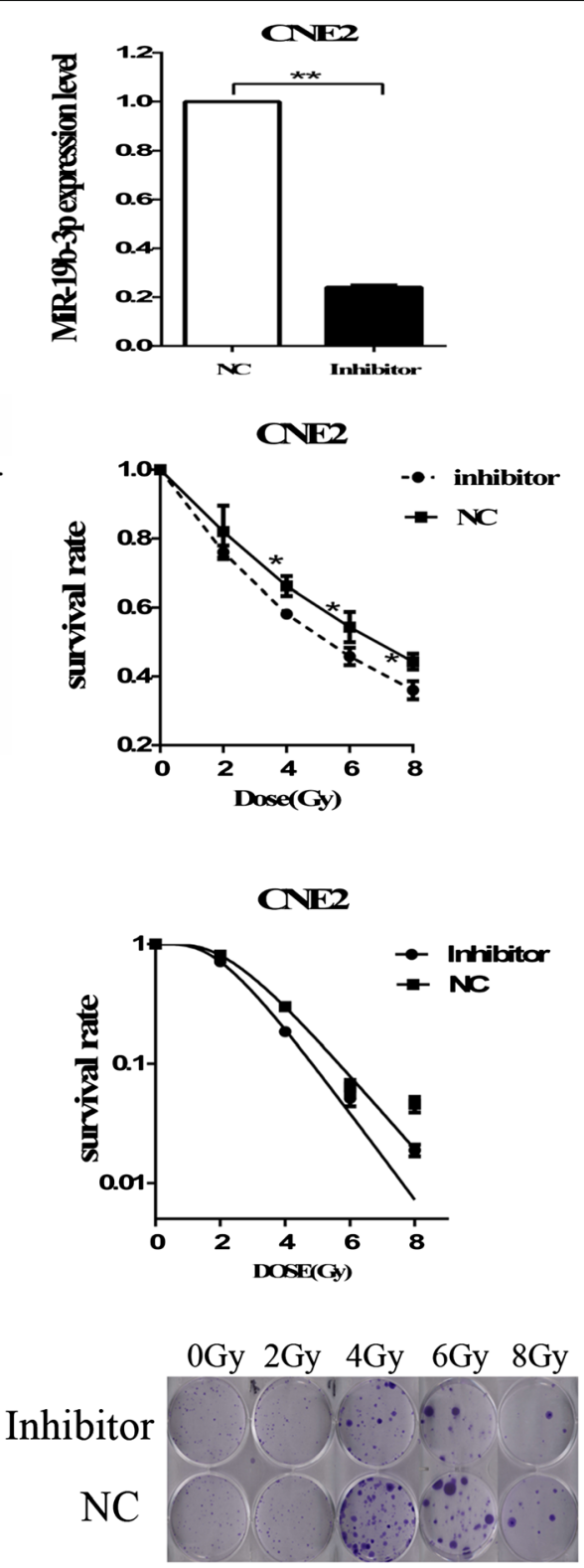

CNE2

Fig. 3 Inhibition of miR-19b-3p increased radiosensitivity of NPC cells. a Downregulated miR-19b-3p expression in transfected cells. b Survival rates of different cell groups after irradiation were examined using CCK-8 assays after 2,4,6,8 Gy irradiation. c Clonogenic survival assay shows that transfection of miR-19b-3p inhibitor increased NPC cell radiosensitivity compared with control groupafter 2,4,6,8 Gy irradiation. d Representative image of colony formation in CNE-1 and CNE-2 cells exposed to different doses of IR

to these 6-8 nt seed sequences of miR-19b-3p were highly conserved among the different species (Fig. 6c).

To further determine whether miR-19b-3p regulates the translation of TNFAIP3, we transfected CNE1 and CNE2 cells with miR-19b-3p mimic and NC mimic, whereas NPC cells were transfected with miR-19b-3p inhibitor and NC inhibitor. qRT-PCR showed that the enhanced levels of miR-19b-3p significantly decreased expression of TNFAIP3 mRNA and that downregulated
miR-19b-3p conversely increased expression of TNFAIP3 mRNA. Western blot analysis revealed that the protein level of TNFAIP3 was markedly reduced in the miR-19b-3p-expressing vector compared with the control vector (Fig. 6d). To further confirm whether the 3'UTR of TNFAIP3 mRNA is a potential functional target of miR-19b-3p, we generated full length TNFAIP3-3' UTR luciferase reporter vectors containing a predicted wild type binding site (TNFAIP3 wt $3^{\prime}$-UTR) or a 


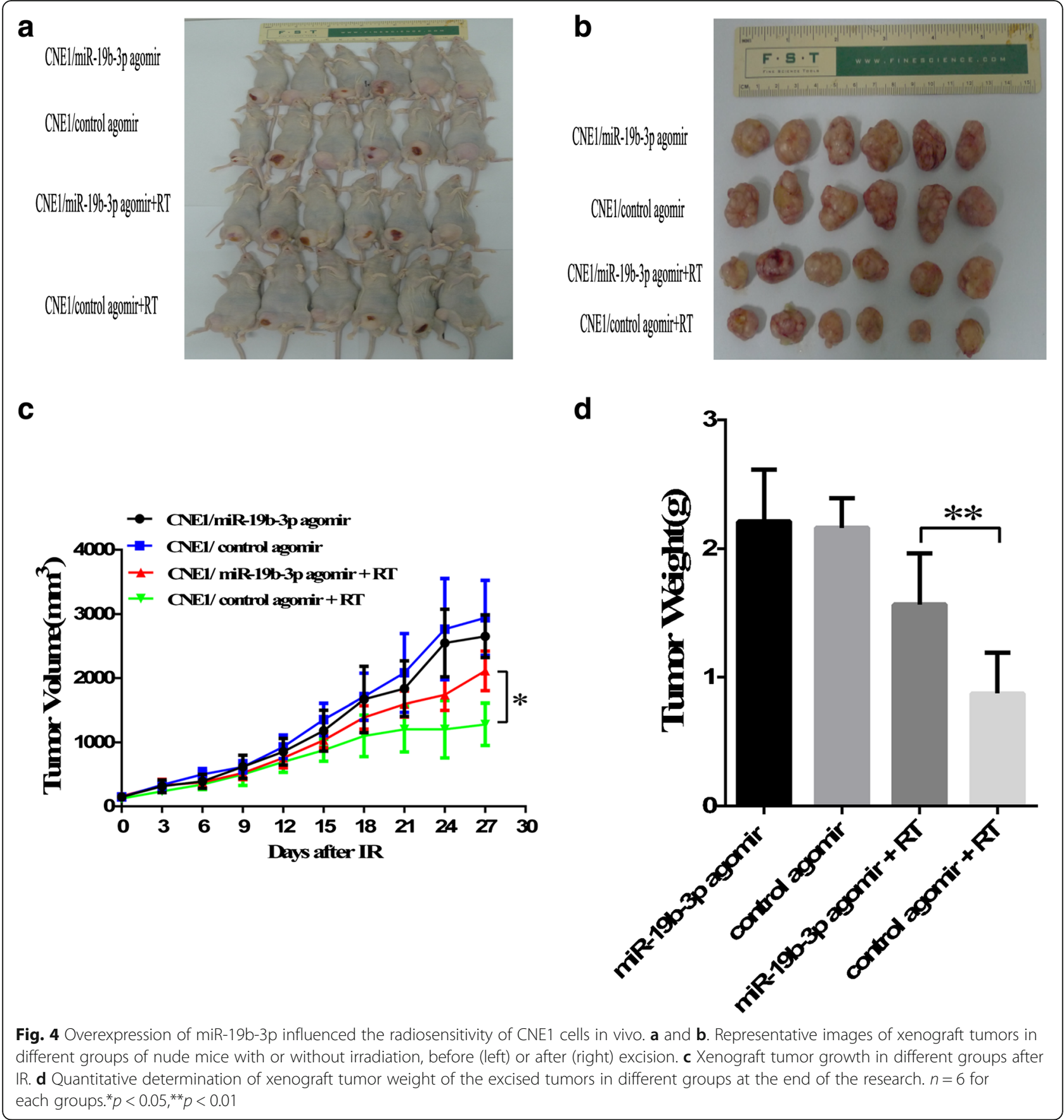

mutated version (TNFAIP3 mut $3^{\prime}$-UTR). These vectors or control pCDNA3.1 vectors were co-transfected with either the miR-19b-3p or the miR-control vector, and luciferase activity was detected after $48 \mathrm{~h}$. Overexpression of the miR-19b-3p reduced the luciferase activity of the TNFAIP3 wt 3'-UTR vector-transfected cells but not that of the TNFAIP3 mut 3'-UTR vector-or of the control pCDNA3.1 vector-transfected cells, whereas the control miR-vector could not significantly change the luciferase activity (Fig. 6e). These results reveal that miR-19b-3p could repress TNFAIP3 translation by targeting a specific 3'UTR mRNA binding sequence.

To clarify the mechanism of TNFAIP3 activity in radioresistance, biological functions of TNFAIP3 were subjected to KEGG Pathway analyses (Fig. 6f), which showed that TNFAIP3 is tightly associated with NF- $\mathrm{KB}$ signaling pathway, which plays an important role in the regulation of radiosensitivity. 


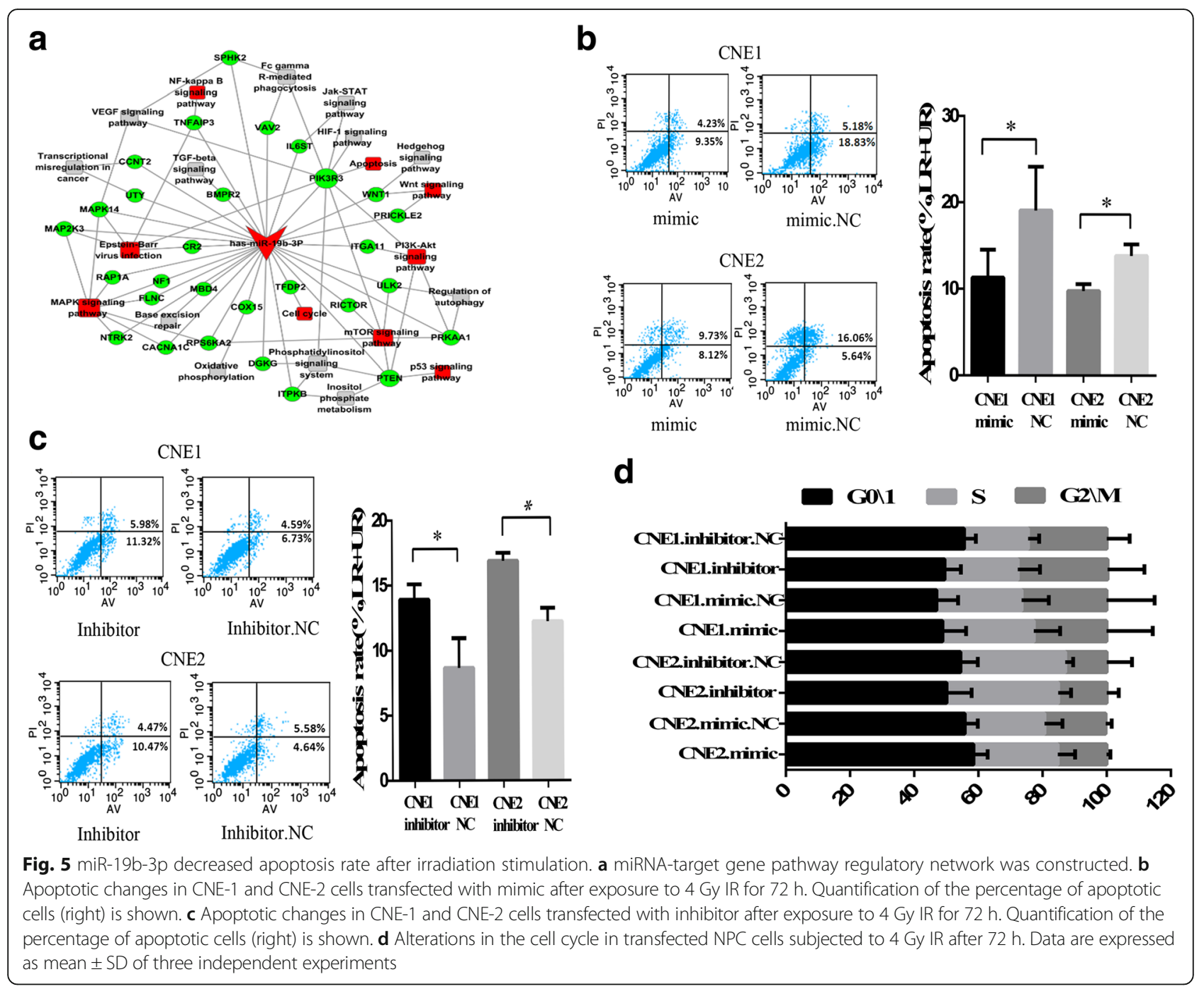

\section{miR-19b-3p decreases radiosensitivity by targeting}

\section{TNFAIP3}

To demonstrate the precise functions of TNFAIP3, we transiently transfected CNE-1 and CNE-2 cells with the siR-TNFAIP3 or siRNA control. A clone survival assay showed that knockout of TNFAIP3 markedly decreased radiosensitivity in the NPC cells [AUC 2.73 (CNE1.siRNA) vs. 2.35 (CNE1.NC), RPF = 1.17 AUC 2.43 (CNE2.siRNA) vs. 2.07 (CNE2.NC), RPF $=1.17 ; p<0.05$ ] (Fig. 7a and b). In addition, the survival rate of CNE1 and CNE2 cells with TNFAIP3 loss was increased after 2,4,6,8 Gy irradiation stimulation, as detected by CCK-8 assay (Fig. 7c). Knockout of TNFAIP3 in CNE1 and CNE2 cells also significantly decreased the percentage of apoptosis after irradiation (Fig. 7d). We then performed colony formation assays by co-transfecting miR-19b-3p inhibitor and siR-TNFAIP3 into NPC cell lines, which showed that decreased expression of TNFAIP3 restored the significant improvement in radiosensitivity by downregulating miR-19b-3p expression
[AUC 3.585 (CNE1.inhibitor plus siRNA) vs 2.857 (CNE1.inhibitor plus siRNA.NC), RPF $=1.25$ AUC 2.99 (CNE1.inhibitor plus siRNA) vs 2.54 (CNE1.inhibitor plus siRNA.NC), RPF $=1.18 ; p<0.05$ ] (Fig. 7e).

miR-19b-3p increased the activity of NF-KB after irradiation stimulation

Having determined that miR-19b-3p was able to regulate NPC radioresistance, its effects on cell apoptosis were analyzed by transfecting CNE1 and CNE2 cells with miR-19b-3p mimic or TNFAIP3 siRNA, respectively, before radiation stimulation. Through bioinformatic analysis, both TNFAIP3 and miR-19b-3p were found to have a correlation with the NF- $\mathrm{kB}$ pathway. Previous studies indicate that activated NF- $\mathrm{B}$ p plays an important role in radioresistance [18]. Therefore, we investigated whether miR-19b-3p mediates NF- $\mathrm{kB}$ signaling pathway by targeting TNFAIP3 and progressively regulated NPC cell radioresponse. In either TNFAIP3 knockdown or 


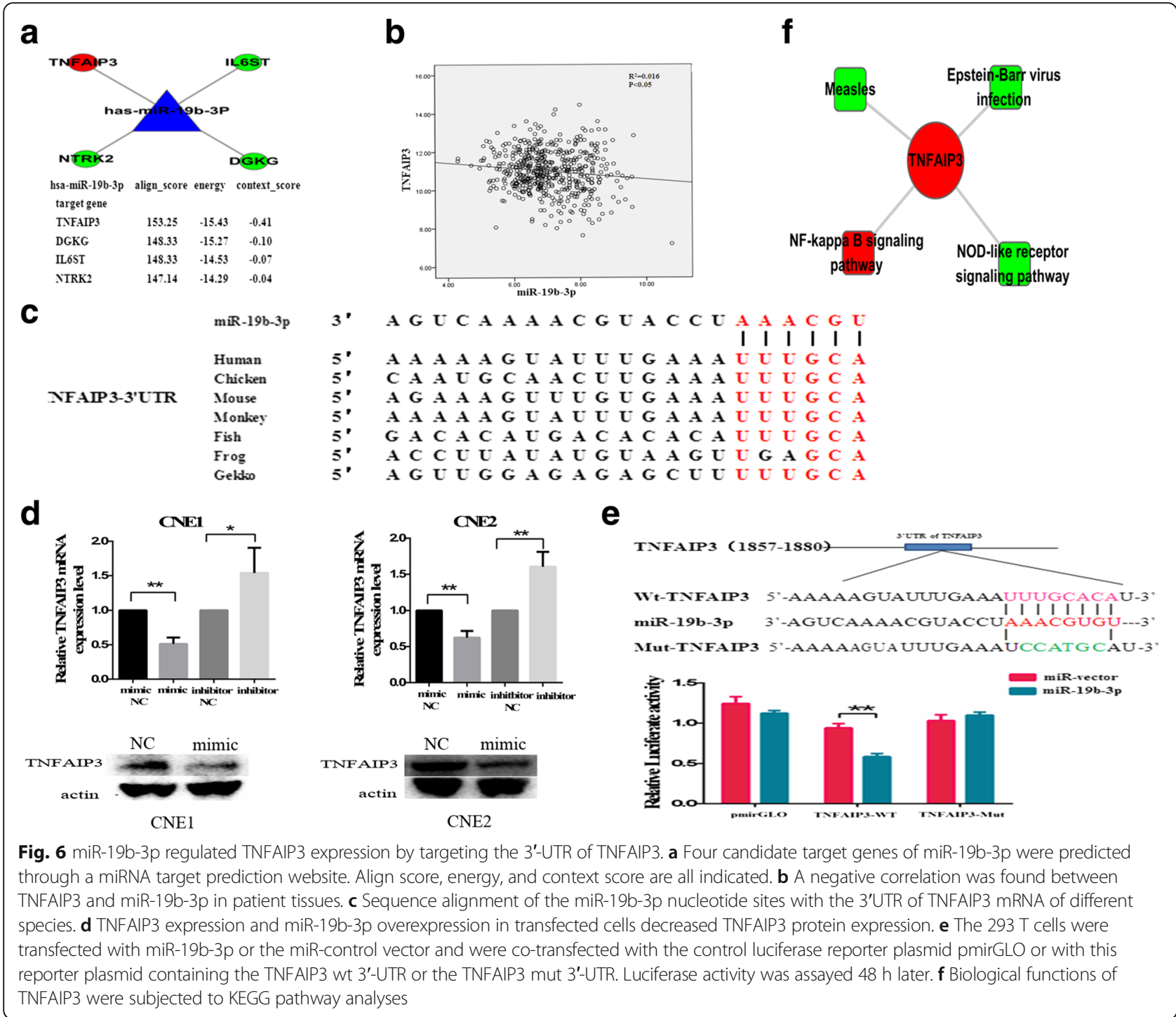

transfection of miR-19b-3p mimic, we obtained similar results after 6 Gy radiation stimulation, as measured by Western blot (Fig. 8). Specifically, p65 level was decreased, and p-p65 level was increased in transfected cell lines compared with control groups. Because NF- $\mathrm{kB}$ is a known transcription factor of $\mathrm{Bcl}-2$, we sought to examine the Bcl-2 expression level by Western blot, which revealed that $\mathrm{Bcl}-2$ level was increased in the transfected cells. We also detected for Bax and ccapase3 levels, which both decreased in the transfected groups compared with the control groups. These studies show that miR-19b-3p can increase NF- $\mathrm{kB}$ activity by targeting TNFAIP3.

\section{Discussion}

With the development of technology, intensity-modulated radiotherapy (IMRT) has improved locoregional control and overall survival of NPC compared with crude two- dimensional radiotherapy techniques and is now the preferred choice of therapy for NPC $[1,19]$. However, NPC cannot be controlled in about $10 \%$ of patients receiving standard dose of radiation therapy; higher doses of radiation therapy produce severe toxicity [1]. Elucidation of the regulatory mechanism underlying radioresistance provides the basis for solving this issue. In the present study, we found that miR-19b-3p regulates the radioresistance of NPC through the NF-kB signaling pathway by targeting TNFAIP3 in vitro.

miRNAs, similar to genes, are classified as tumorcausing (called "oncomiRs") or tumor-suppressive ("tumor-suppressive miRNAs") based on their roles in cancer [2]. miR-19b has been shown to have a preponderant role in oncogenicity, and is an essential regulator related to tumor malignant biobehavior. miR-19b can enhance migration and invasion of lung cancer cells [20] and is associated with drug tolerance [21]. In cellular 


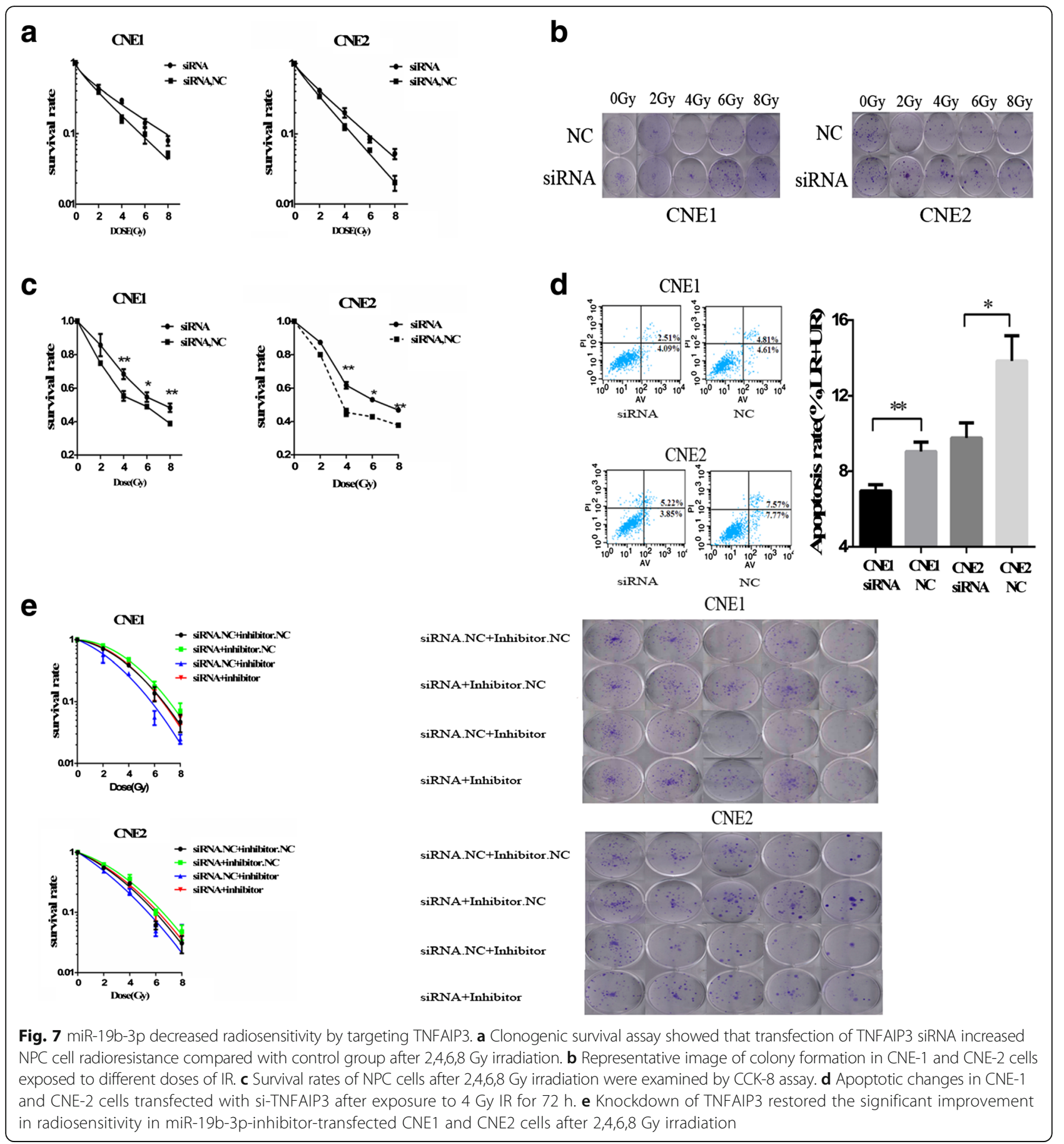

proliferation, however, miR-19b elicits different effects in different tissues; miR-19b accelerates melanoma cell proliferation, whereas it elicits the opposite effect in hepatic stellate cell $[12,22]$. However, the function of miR-19b$3 p$ in radioresistance is hardly studied. In the present study, we found that miR-19b-3p is upregulated in NPC and is associated with worse patient prognosis. Our data revealed that miR-19b-3p contributes to radioresistance in vitro and in vivo. miRNAs exert significant biological functions by regulating various target genes [23]. Then, via the miRNA target prediction program, TNFAIP3 is predicted as a potential target with the highest predictive value for miR-19b-3p. We further verified that miR-19b$3 p$ regulates TNFAIP3 expression level by targeting the 3 '-UTR of TNFAIP3 mRNA.

TNFAIP3 is widely considered as an important regulator of inflammation and immunity, and plays a key role in the modulation of immune response to pathogens as 


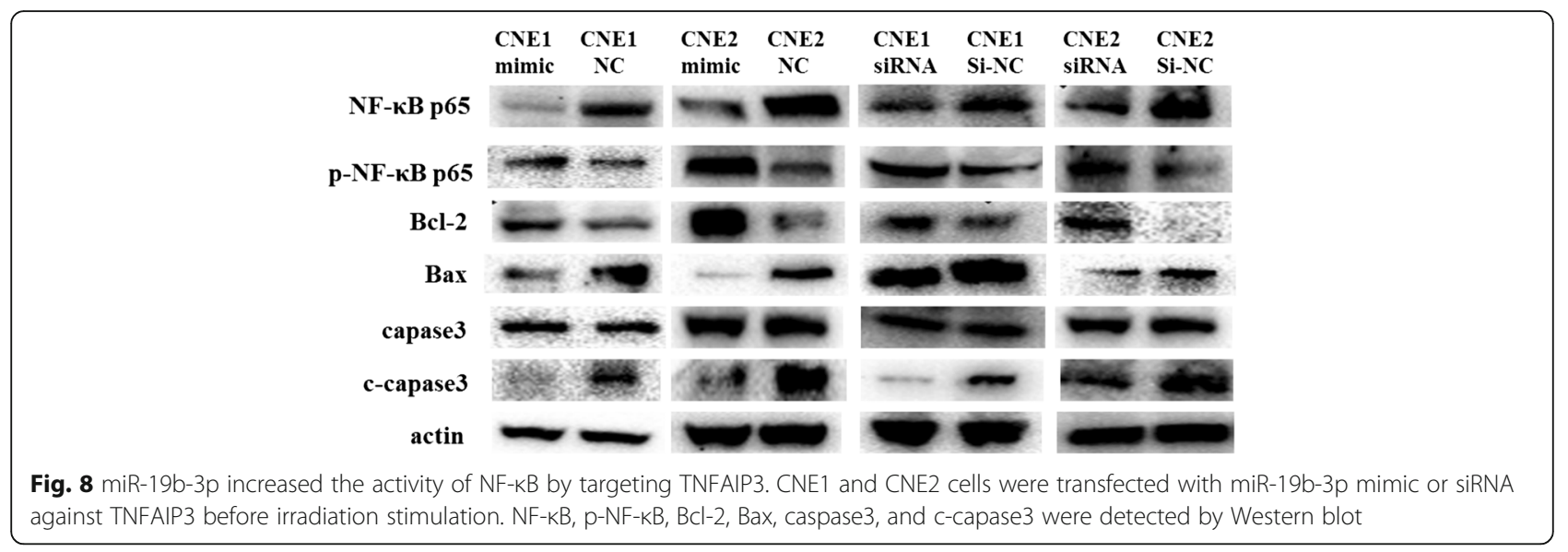

well as the development of inflammatory and autoimmune disease $[24,25]$. Moreover, TNFAIP3 is now accepted as a disease susceptibility gene that may serve as a predictive biomarker [26]. However, the potential roles of TNFAIP3 in regulation of radiosensitivity are rarely studied. Our data demonstrated that knockout of TNFAIP3 can decrease NPC cell radiosensitivity. Furthermore, TNFAIP3 knockdown abolished radiosensitization induced by transfection of miR-19b-3p inhibitor in NPC cells. Overall, miR-19b-3p regulated nasopharyngeal carcinoma radioresistance by targeting TNFAIP3.

Apoptosis is an important mechanism for the regulation of sensitivity to radiation [9, 27]. We found that overexpression of miR-19b-3p or knockout of TNFAIP3 decreased apoptosis rates of NPC cell lines after irradiation. Using bioinformatic analyses, we found that both miR-19b-3p and TNFAIP3 have a common apoptotic pathway-the NF- $\mathrm{kB}$ signaling pathway. Ionizing radiation is known to cause DNA
DSBs and leads to lethal injury [9, 28]. IR-induced DSBs trigger a DNA damage response with $N F-\kappa B$ activation as the antiapoptotic factor in most cell types [29-32]. Thus, through the inhibition of NF$\kappa \mathrm{B}$ activation, radiosensitivity can be increased [33]. Previous studies have demonstrated that TNFAIP3 acts as a repressor that downregulates NF- $\mathrm{kB}$ activity. Indeed, we found that NF- $\mathrm{KB}$ activity was enhanced after radiation stimulation by the knockout of TNFAIP3. Furthermore, we confirmed that NF- $\mathrm{BB}$ activity improved after radiation stimulation by overexpressing miR-19b-3p. To further investigate the possibility of the mitochondrial signaling pathway as a molecular pathway for miR-19b-3p, the expressions of Bcl-2, Bax, capase3, and c-capase3 markers were examined. Our data revealed similar protein results from the NPC cell lines after either TNFAIP3 knockdown or transfection of miR-19b-3p mimic. miR-19b$3 p$ increased the radioresistance of NPC by increasing NF- $\mathrm{BB}$ activity through TNFAIP3 targeting (Fig. 9).

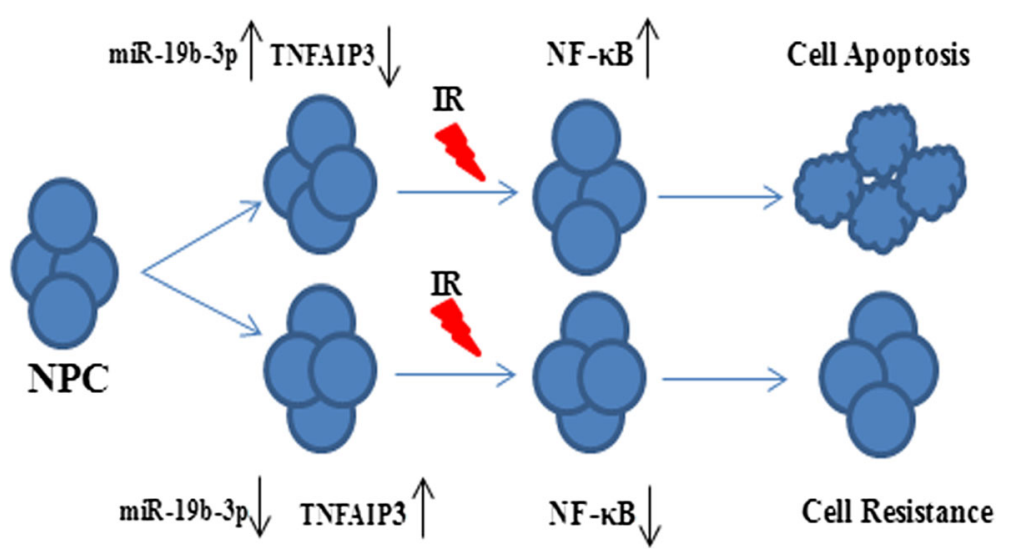

Fig. 9 miR-19b-3p increased the radioresistance of NPC 


\section{Conclusions}

We revealed that miR-19b-3p is upregulated in NPC and serves as a regulator of NF- $\mathrm{BB}$ activity by targeting TNFAIP3. The newly identified miR-19b-3p/TNFAIP3/ NF- $\mathrm{KB}$ axis sheds light on a novel molecular mechanism for NPC cell radioresistance, indicating that miR-19b-3p is a valuable biomarker and a promising therapeutic target for the management of NPC.

\section{Additional files}

\section{Additional file 1: Sample ID. (XLSX $11 \mathrm{~kb})$}

Additional file 2: Correlation between the expression of miR-19b-3p and clinical features. (DOCX $18 \mathrm{~kb}$ )

Additional file 3: Sample ID. (XLSX $26 \mathrm{~kb}$ )

\section{Abbreviations}

AUC: Area under the curve; Cl: Confidence interval; DSBs: DNA double-strand breaks; HR: Hazard ratio; IR: lonizing radiation; MID: Mean inactivation dose; miRNA: MicroRNA; NPC: Nasopharyngeal carcinoma; ROC: Receiver operating characteristic; RPF: Radiation protection factor

\section{Acknowledgments}

This study was supported by the JiangSu Clinical Medicine Science and Technology Special Fund (No. BL2014091).

\section{Funding}

JiangSu Clinical Medicine Science and Technology Special Fund (No. BL2014091).

\section{Availability of data and materials}

1. The datasets generated during and/or analysed during the current study are availablein

1) the [GEO] repository (www.ncbi.n/m.nih.gov/geo/)

2) the [TCGA] repository: (https:// tcga-data.nci.nih.gov and https:// genome-cancer.ucsc.edu)

2. The datasets during and/or analysed during the current study available from the corresponding author on reasonable request.

\section{Authors' contributions}

$\mathrm{XH}$ and $\mathrm{TH}$ conceived and designed the experiments. TH performed the experiments. JW and LY coordinated the research and analyzed the data. TH and LY wrote the manuscript. J-JG, DC, H-LY, KD and NZ supported the experiments and helped to draft the manuscript. J-ZW and M-YD, L-XQ, Z-WL provided the financial support and supervised laboratorial processes. All authors read and approved the final manuscript.

\section{Competing interests}

The authors declare that they have no competing interests.

\section{Consent for publication}

Not applicable.

\section{Ethics approval}

Our animal protocols were approved by the Institutional Animal Care and Use Committee of Nanjing Medical University (Nanjing, China). All animal experiments are in accordance with the guidelines of humane use and care of laboratory animals for biomedical research published by National Institutes of Health.

\section{Author details}

'The Fourth Clinical School of Nanjing Medical University, Nanjing, Jiangsu, China. ${ }^{2}$ Department of Radiation Oncology, Nanjing Medical University Affiliated Cancer Hospital, Cancer Institute of Jiangsu Province, 42 Bai Zi Ting Road, Nanjing, Jiangsu 210000, China. ${ }^{3}$ Research Center of Clinical Oncology,
Nanjing Medical University Affiliated Cancer Hospital, Cancer Institute of Jiangsu Province, Nanjing, Jiangsu, China.

Received: 2 August 2016 Accepted: 22 November 2016

Published online: 05 December 2016

\section{References}

1. Chua ML, Wee JT, Hui EP, Chan AT. Nasopharyngeal carcinoma. Lancet. 2016;387:1012-24

2. Wang S, Zhang R, Claret FX, Yang H. Involvement of microRNA-24 and DNA methylation in resistance of nasopharyngeal carcinoma to ionizing radiation. Mol Cancer Ther. 2014;13:3163-74.

3. Gandellini $P$, Rancati $T$, Valdagni R, Zaffaroni N. miRNAs in tumor radiation response: bystanders or participants? Trends Mol Med. 2014;20:529-39.

4. Wang $F$, Lu J, Peng $X$, et al. Integrated analysis of microRNA regulatory network in nasopharyngeal carcinoma with deep sequencing. J Exp Clin Cancer Res. 2016;35:17.

5. Guo P, Lan J, Ge J, et al. MiR-26a enhances the radiosensitivity of glioblastoma multiforme cells through targeting of ataxia-telangiectasia mutated. Exp Cell Res. 2014:320:200-8.

6. Liu S, Pan X, Yang Q, et al. MicroRNA-18a enhances the radiosensitivity of cervical cancer cells by promoting radiation-induced apoptosis. Oncol Rep. 2015;33:2853-62.

7. Wang $P$, Zou F, Zhang $X$, et al. MicroRNA-21 negatively regulates $C d c 25 A$ and cell cycle progression in colon cancer cells. Cancer Res. 2009;69:8157-65.

8. Zhang $X, N g$ WL, Wang $P$, et al. MicroRNA-21 modulates the levels of reactive oxygen species by targeting SOD3 and TNFalpha. Cancer Res. 2012; 72:4707-13

9. Tang Y, Cui Y, Li Z, et al. Radiation-induced miR-208a increases the proliferation and radioresistance by targeting p21 in human lung cancer cells. J Exp Clin Cancer Res. 2016;35:7.

10. Hu. MicroRNA-181a sensitizes human malignant glioma U87MG cells to radiation by targeting BCl-2. Oncology Reports. 2010;23:997-1003.

11. Tang $Y$, Zhang YC, Chen $Y$, et al. The role of miR-19b in the inhibition of endothelial cell apoptosis and its relationship with coronary artery disease. Sci Rep. 2015;5:15132.

12. Ohira T, Naohiro $S$, Nakayama $Y$, et al. miR-19b regulates hTERT mRNA expression through targeting PITX1 mRNA in melanoma cells. Sci Rep. 2015;5:8201.

13. Zhang JX, Qian D, Wang FW, et al. MicroRNA-29C enhances the sensitivities of human nasopharyngeal carcinoma to cisplatin-based chemotherapy and radiotherapy. Cancer Lett. 2013;329:91-8.

14. Li G, Liu Y, Su Z, et al. MicroRNA-324-3p regulates nasopharyngeal carcinoma radioresistance by directly targeting WNT2B. Eur J Cancer. 2013; 49:2596-607.

15. Li G, Wang Y, Liu Y, et al. miR-185-3p regulates nasopharyngeal carcinoma radioresistance by targeting WNT2B in vitro. Cancer Sci. 2014;105:1560-8.

16. Qu JQ, Yi HM, Ye X, et al. MiR-23a sensitizes nasopharyngeal carcinoma to irradiation by targeting IL-8/Stat3 pathway. Oncotarget. 2015;6:28341-56.

17. Yang SH, Kuo TC, Wu H, et al. Perspectives on the combination of radiotherapy and targeted therapy with DNA repair inhibitors in the treatment of pancreatic cancer. World J Gastroenterol. 2016;22:7275-88,

18. Deorukhkar A, Krishnan S. Targeting inflammatory pathways for tumor radiosensitization. Biochem Pharmacol. 2010;80:1904-14.

19. Duru N, Gernapudi $R$, Zhang $Y$, et al. NRF2/miR-140 signaling confers radioprotection to human lung fibroblasts. Cancer Lett. 2015;369:184-91.

20. Li J, Yang S, Yan W, et al. MicroRNA-19 triggers epithelial-mesenchymal transition of lung cancer cells accompanied by growth inhibition. Lab Invest. 2015;95:1056-70.

21. Kurokawa K, Tanahashi T, lima T, et al. Role of miR-19b and its target mRNAs in 5-fluorouracil resistance in colon cancer cells. J Gastroenterol. 2012;47: 883-95.

22. Ge S, Xie J, Liu F, et al. MicroRNA-19b reduces hepatic stellate cell proliferation by targeting GRB2 in hepatic fibrosis models in vivo and in vitro as part of the inhibitory effect of estradiol. J Cell Biochem. 2015;116: 2455-64.

23. Xiao H, Xiao W, Cao J, et al. miR-206 functions as a novel cell cycle regulator and tumor suppressor in clear-cell renal cell carcinoma. Cancer Lett. 2016;374:107-16.

24. Catrysse L, Vereecke L, Beyaert R, van Loo G. A20 in inflammation and autoimmunity. Trends Immunol. 2014;35:22-31. 
25. Wertz IE, Newton K, Seshasayee D, et al. Phosphorylation and linear ubiquitin direct A20 inhibition of inflammation. Nature. 2015;528:370-5.

26. Adrianto I, Wen F, Templeton A, et al. Association of a functional variant downstream of TNFAIP3 with systemic lupus erythematosus. Nat Genet. 2011:43:253-8.

27. Yan Y, Xu Z, Dai S, et al. Targeting autophagy to sensitive glioma to temozolomide treatment. J Exp Clin Cancer Res. 2016;35:23.

28. Dong $Y$, Shen $X$, He M, et al. Activation of the JNK-c-Jun pathway in response to irradiation facilitates Fas ligand secretion in hepatoma cells and increases hepatocyte injury. J Exp Clin Cancer Res. 2016;35:114.

29. Habraken Y, Piette J. NF-kappaB activation by double-strand breaks. Biochem Pharmacol. 2006;72:1132-41.

30. Tang Y, Geng Y, Luo J, et al. Downregulation of ubiquitin inhibits the proliferation and radioresistance of non-small cell lung cancer cells in vitro and in vivo. Sci Rep. 2015:5:9476.

31. Thangavel C, Boopathi E, Ciment S, et al. The retinoblastoma tumor suppressor modulates DNA repair and radioresponsiveness. Clin Cancer Res. 2014;20:5468-82.

32. Hunter JE, Willmore E, Irving JA, et al. NF-kappaB mediates radio-sensitization by the PARP-1 inhibitor, AG-014699. Oncogene. 2012;31:251-64.

33. Chen $Y$, Tang $Q$, Wu J, et al. Inactivation of PI3-K/Akt and reduction of SP1 and p65 expression increase the effect of solamargine on suppressing EP4 expression in human lung cancer cells. J Exp Clin Cancer Res. 2015;34:154.

\section{Submit your next manuscript to BioMed Central} and we will help you at every step:

- We accept pre-submission inquiries

- Our selector tool helps you to find the most relevant journal

- We provide round the clock customer support

- Convenient online submission

- Thorough peer review

- Inclusion in PubMed and all major indexing services

- Maximum visibility for your research

Submit your manuscript at www.biomedcentral.com/submit

) Biomed Central 